\title{
Das Rad neu erfinden?
}

\section{Ansätze und Möglichkeiten transdisziplinärer ökologischer Wirtschaftsfor- schung standen im Zentrum der Ausgabe 1/98 von Ökologisches Wirtschaften. Der folgende Beitrag nimmt kritisch dazu Stellung und erinnert insbesondere an bereits reichlich vorliegende praktische Erfahrungen.}

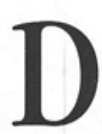
Von Stefan Zundel ie Wissenschaft sollte auch praktische Antworten auf drängende Probleme liefern. Das ist ein Anspruch der Autoren dieses Schwerpunktes, den ich teile. Dennoch bin ich unzufrieden mit den vorgetragenen Argumenten. Die ökologische Herausforderung ist nicht die erste Gelegenheit, bei der reale Phänomene einer ausdifferenzierten Wissenschaft Syntheseleistungen abverlangen, und sie wird wohl auch nicht die letzte Gelegenheit dieser Art sein. Man denke nur an die großen gesellschaftstheoretischen Entwürfe, die wohl kaum umstandslos der Ökonomie, der Soziologie oder der Psychologie unterzuordnen sind. Deshalb hat es mich gewundert, daß ein Teil der unter dem Schwerpunkt versammelten Autoren die Attitüde eines völlig neuen Programms pflegt.

Dieser Eindruck des Unbehagens wird noch verstärkt: $\mathrm{Zu}$ den ersten wissenschaftlichen „Slogans“, mit denen ich zu Beginn meiner eigenen wissenschaftlichen Laufbahn auf dem Gebiet der ökologischen Wirtschaftsforschung konfrontiert wurde, gehörte die Formel, wonach Ökologie ein Querschnittsthema sei. Und ich bin mir ziemlich sicher, daß dieser Ausdruck schon damals nicht sehr originell war. Diese Formel hört sich vielleicht nicht so gut an wie Transdisziplinarität, gemeint ist aber doch wohl etwas sehr ähnliches. Vor diesem Hintergrund kann ich nicht verstehen, warum

\section{Perspektiven des Öko-Audit}

Sowie ein Spezial: ÖkonomischÖkologische Komplexität sind die Themen des Informationsdienstes

Ökologisches Wirtschaften 3/98

Wenn Sie potentielle Beiträge haben, wenden Sie sich bitte an die Redaktion! die Autoren des Schwerpunktes mit der Ausnahme von Martin Jänicke so gut wie gar nicht die mittlerweile umfangreichen Erfahrungen mit disziplinübergreifenden Projekten reflektieren, die im Einzugsbereich der ökologischen Wirtschaftsforschung auf nationaler und auf internationaler Ebene aus den letzten Jahren vorliegen.

Einige dieser Erfahrungen in Kürze: Disziplin übergreifende Zusammenarbeit ist ein sehr zeitaufwendiger Prozeß, weil sehr viel Kraft in die wechselseitige Verständigungsbemühung gesteckt werden muß. Deshalb bescheiden sich aus pragmatischen Gründen viele Wissenschaftsprojekte dieser Art auf eine Art ,Andockmodell": die einzelnen Disziplinen liefern eigenständige disziplintypische Module im Rahmen eines gemeinsam vereinbarten Analyseschemas, ein integrierter theoretischer Ansatz liegt jedoch nicht vor. Das dürfte kein Zufall sein, sondern muß seinerseits methodisch reflektiert und erklärt werden.

\section{Vielzahl von Ansätzen}

Aber selbst wenn der Anspruch auf einen transdisziplinären Ansatz nicht nur erhoben, sondern real auch eingelöst wird: Wie jede theoretische Leistung müssen auch integrierende disziplin-übergreifende Ansätze die komplexe Realität zu einem Modell oder doch wenigstens einer Heuristik reduzieren, wenn sie nicht bei der trivialen Erkenntnis enden wollen, daß alles irgendwie mit allem zusammenhängt und das ist herzlich uninteressant. Auf diese Weise entsteht i.d.R. eine neue Theorie und, wenn der Gegenstand der Theorie das hergibt, manchmal auch eine neue Wissenschaftsdisziplin. Im Bereich der ökologischen Forschung - der Zusatz „Wirtschaft“ will angesichts des transdisziplinären Anspruchs nicht so richtig passen - gibt es da eine Menge Konkurrenz um den richtigen Weg. Dazu zählt nicht nur der Syndrom-Ansatz des WBGU oder der Bedürfnisfeldansatz der Enquete, wenn man hier über- haupt von einem wissenschaftlichen Ansatz sprechen kann. Neben den genannten Ansätzen sei z.B. noch auf systemtheoretische Syntheseversuche des Ökologieproblems, kommunikationstheoretische Ansätze und solche, die sich auf psychologische Modelle und Vorstellungen des Menschen stïtzen, hingewiesen. Sie alle beanspruchen ebenfalls, jenen Zipfel des Tischtuches erfaßt zu haben, mit dem das ganze Ökologieproblem theoretisch und praktisch in Bewegung gesetzt werden kann. Alles das locker links und rechts liegen zu lassen, und dann die Frage ,Wie ist Transdisziplinarität möglich?" nur noch am Bedürfnisfeldansatz der Enquete und dem Syndromansatz des WBGU zu debattieren, zeugt entweder von einiger Chuzpe oder von beträchtlicher Theorievergessenheit.

Ein anderer Beleg illustriert den gleichen Gedanken: Wenn die Autoren des Ansatzes der Transdisziplinarität einmal die programmatischen Bemühungen, die am Anfang der Gründung des IÖW standen und im IÖW/vöW-Informationsdienst, dem Vorläuferorgan des Ökologischen Wirtschaften, veröffentlicht wurden, nachgelesen hätten, dann hätten sie, wenn auch in einer anderen sprachlichen Verkleidung, fast jeden Gedanken wiedergefunden, den sie heute als neue Erkenntnis einbringen. Das Problem der Inter- oder Transdisziplinarität ist doch nicht, daß irgend jemand heute ernsthaft bestreiten würde, daß das wichtig ist. Das Problem steckt in der Frage, warum sich die disziplinübergreifenden Ansätze - beispielsweise auch die, an denen das IÖW beteiligt war - in den vergangenen zehn Jahren so schwer taten und das immer noch tun.

Wer sich unter diesen Umständen nicht die Mühe macht, sich mit der Geschichte der disziplinübergreifenden Projekte auseinanderzusetzen, wird in der Zukunft die Fehler wiederholen müssen, die in der Vergangenheit schon einmal gemacht wurden.

\section{Der Autor}

Dr. Stefan Zundel ist Professor für Öffentliche Finanzwirtschaft, Energie und Umwelt im Fachbereich Wirtschaftswissenschaften der Fachhochschule Lausitz. Er ist Vorstandsmitglied und ehemaliger Geschäftsführer des IÖW.

Kontakt: FH Lausitz, Großenhainer Straße 57, 01968 Senftenberg, Tel. 03573/ 85733, Fax 03573/ 85709, E-mail: zundel@fh-lausitz.de 
(c) 20I0 Authors; licensee IÖW and oekom verlag. This is an article distributed under the terms of the Creative Commons Attribution Non-Commercial No Derivates License (http://creativecommons.org/licenses/by-nc-nd/3.o/), which permits unrestricted use, distribution, and reproduction in any medium, provided the original work is properly cited. 